\title{
Coupling Fault Feature Extraction Method Based on Bivariate Empirical Mode Decomposition and Full Spectrum for Rotating Machinery
}

\author{
Rong Jia $\mathbb{D}^{\mathbb{D}},{ }^{1}$ Fuqi Ma $\mathbb{D}^{1},{ }^{1}$ Hua Wu, ${ }^{1}$ Xingqi Luo, ${ }^{1}$ and Xiping $\mathrm{Ma}^{2}$ \\ ${ }^{1}$ State Key Laboratory Base of Eco-Hydraulic Engineering in Arid Area, Xian University of Technology, Xian, Shanxi 710048, China \\ ${ }^{2}$ Gansu Province Electric Power Research Institute, Lanzhou, Gansu 730050, China \\ Correspondence should be addressed to Fuqi Ma; 18392647176@163.com
}

Received 14 September 2017; Revised 21 January 2018; Accepted 4 February 2018; Published 7 March 2018

Academic Editor: Frederic Kratz

Copyright (C) 2018 Rong Jia et al. This is an open access article distributed under the Creative Commons Attribution License, which permits unrestricted use, distribution, and reproduction in any medium, provided the original work is properly cited.

\begin{abstract}
To accurately extract the fault characteristics of vibration signals of rotating machinery is of great significance to the unit online monitoring and evaluation. However, because the current feature extraction methods are mainly for single channel, the results of feature extraction are often inaccurate. To this end, a coupling fault feature extraction method based on bivariate empirical mode decomposition (BEMD) and full spectrum is proposed for rotating machinery. Firstly, the two-dimensional orthogonal signal obtained by orthogonal sampling technique is decomposed by bivariate empirical mode decomposition to obtain the intrinsic mode function with phase information. In order to obtain the sensitive modal components, the sensitivity coefficients are constructed on the basis of mutual information. Then, the sensitivity coefficient of each intrinsic mode function is calculated, and the intrinsic mode function with the larger sensitive coefficient is selected as the sensitive component. Finally, the full spectrum of the sensitive component is obtained using the full vector envelope technique, so as to get a comprehensive and accurate characteristic component. The results of simulations experiment and an application example show that this method can extract the fault characteristic component of the rotating machinery comprehensively and accurately. It is of great significance to realize the accurate diagnosis of coupling faults of rotating machinery.
\end{abstract}

\section{Introduction}

Rotating machinery is one of the key parts of large mechanical equipment, such as hydraulic turbine, steam turbine, and wind turbine, which have found wide application in various industrial fields [1]. Due to the influence of load, damping, friction, and other factors, the rotating machinery usually exhibits complex dynamic behavior, which is related to nonstationarity and nonlinearity [2]. And the vibration signals of rotating machinery often show coupling. In order to ensure the safe and reliable operation of the equipment, the equipment condition monitoring and fault diagnosis technology needs to have higher precision. Whether it can accurately extract the characteristic signal of coupling fault is the key to the condition monitoring and fault diagnosis of rotating machinery $[3,4]$.
At present, many scholars have done some research on the feature extraction of rotating machinery fault signal, especially in the matter of signal processing methods. The main methods are wavelet transform (WT) [5, 6], Hilbert-Huang transform, empirical mode decomposition (EMD) [7, 8], variational mode decomposition (VMD) $[9,10]$, and so on, and the characteristics of different fault feature extraction methods are shown in Table 1. Wavelet transform is the improvement of the Fourier transform and has a certain ability to deal with nonlinear signals. However, its essence is the inner product principle characteristic waveform signal decomposition based on basis function, but there exists the problem of basis functions selection, and the different vibration will show the characteristics of different waveforms [11, 12]. Therefore, the adaptive ability of wavelet transform is poor. EMD has strong adaptability and good local analysis 
TABLE 1: The characteristics of different fault feature extraction method.

\begin{tabular}{lc}
\hline Method & Characteristic \\
WT & $\begin{array}{c}\text { The choice problem for basis functions } \\
\text { exists and the different vibration will } \\
\text { show the characteristics of different } \\
\text { waveforms. }\end{array}$ \\
\hline EMD & $\begin{array}{c}\text { It lacks rigorous theoretical basis and } \\
\text { there is a serious phenomenon of modal } \\
\text { aliasing in the decomposition process. }\end{array}$ \\
\hline VMD & $\begin{array}{c}\text { It solves the problem of mode mixing in } \\
\text { EMD, but there are parameter } \\
\text { optimization problems in VMD. }\end{array}$ \\
\hline BEMD & $\begin{array}{c}\text { It can detect synchronous features } \\
\text { contained in the two-dimensional signal } \\
\text { and comprehensively extract the } \\
\text { information of fault signal. }\end{array}$ \\
\hline
\end{tabular}

ability and has been widely used in the field of fault diagnosis. However, the EMD method lacks rigorous theoretical basis, and there is a serious phenomenon of modal aliasing in the decomposition process $[13,14]$. Variational mode decomposition (VMD) is a new adaptive signal decomposition method, which has a solid theoretical foundation and can solve the problem of mode mixing in EMD, but there are parameter optimization problems in VMD [15].

However, due to the complex mechanism of the fault of rotating machinery, as well as the vibration signal being often nonlinear, the vibration signals in different directions may represent different fault characteristic information [16]. Through single-channel signal feature extraction and fault diagnosis, it often leads to misjudgment and omission $[17,18]$. Therefore, it is more accurate to collect the signal of multiple channels, through the effective information fusion. Therefore, taking into account the rotating machinery, which generally has two sensors in horizontal and vertical directions, this paper utilizes bivariate empirical mode decomposition (BEMD) to decompose the signal collected from two directions.

In 2007, bivariate empirical mode decomposition (BEMD) was firstly introduced by Rilling et al. [19]. It is not limited to single direction processing of real valued signals, and it can analyze complex signals consisting of two orthogonal directions containing phase information and synchronization $[20,21]$.

Molla et al. [22] applied bivariate empirical mode decomposition to decompose complex climate signals into simple components and then combined BEMD with the classical FFT spectrum to clearly observe the climate change. Then Yang et al. [23] used bivariate empirical mode decomposition on condition monitoring for wind turbine; this method can overcome the problem of inability to carry out an information fusion function in EMD, which is of importance to reach a reliable condition monitoring conclusion.

The joint information between different sensors such as phase information and synchronization is of significance for rotating machinery condition to evaluate [24]. The signal obtained from multiple sensors is evaluated to contribute to the effective extraction of fault information, so it has received increasing attention in the past years. Okatan et al. [25] use the normalized matrix of the spectral norm to propose the new Kalman filter and propose a sensor information fusion Kalman filter test method, which improves the fusion accuracy. Liu et al. [26] proposed a fuzzy fault measurement method and a fuzzy integral information fusion method for rotating machinery. By using multiple classifiers, fuzzy measures, and fuzzy integral theory, the diagnosis results are fused and the final diagnosis results are obtained. The full spectrum is a fault diagnosis method based on homologous information fusion technology, which can acquire more comprehensive fault characteristics and reveal the direction of the vibration relative to the direction of the vibration of rotating machinery $[24,27]$.

In this paper, we proposed a coupling fault feature extraction method based on bivariate empirical mode decomposition and full spectrum for rotating machinery. In order to suppress noise interference in the signal component, the sensitivity coefficients are constructed on the basis of mutual information and through the sensitive coefficient to extract unique IMFs of the signal. The specific arrangement of this paper is organized as follows. A review on bivariate empirical mode decomposition (BEMD) is illustrated in Section 2. Section 3 describes the sensitive feature extraction based on mutual information. Section 4 gives brief introductions of full spectrum. Section 5 describes the coupling fault feature extraction method based on BEMD and full spectrum. Section 6 applies the proposed method to both simulated data and experimental data obtained from the turbine guide bearing. Conclusions come in Section 7.

\section{Bivariate Empirical Mode Decomposition}

The essence of bivariate empirical mode decomposition (BEMD) is to decompose a two-dimensional signal adaptively into intrinsic mode components with physical meaning. The modal component obtained from the decomposition is a series of single component signals from high frequency to low frequency in two directions. This paper takes Algorithm II in [19] to perform BEMD. For a two-dimensional signal $x(t)$, the basic decomposition process is as follows.

Step 1. Determine the direction of projection $\varphi_{k}=2 k \pi / N$, where $1 \leq k \leq N$.

Step 2. The two-dimensional signal $x(t)$ is projected onto the $\phi_{k}$.

$$
p_{\varphi_{k}}(t)=\operatorname{Re}\left(e^{-\varphi_{k}} x(t)\right) .
$$

Step 3. Extract the corresponding moment for the local maximum of $X\left\{t_{j}^{k}\right\}$; then the set $\left\{t_{j}^{k}, e^{i \varphi_{k}} p_{j}^{k}\right\}$ is interpolated. Get the maximum envelope $e_{\varphi_{k}}^{\prime}(t)$ in the direction $\varphi_{k}$.

Step 4. Calculate the mean of the maximum envelope $e_{\varphi_{k}}^{\prime}(t)$ in each direction. 


$$
m(t)=\frac{2}{N} \sum_{k=1}^{N} e_{\varphi_{k}}^{\prime}(t) .
$$

Step 5. Similar to the EMD decomposition process, the residual component is calculated:

$$
S(t)=x(t)-m(t) .
$$

Determine whether the $S(t)$ meets the requirements of IMF. If satisfied, proceed to Step 6, and if not, repeat Steps 2-6 until $S(t)$ satisfies the conditions of the intrinsic mode function IMF.

Step 6. Record the resulting IMF and remove it from the original signal. And get IMF1 as $c_{1}(t)=h(t)$, residual component as

$$
r_{1}(t)=x(t)-c_{1}(t)
$$

Step 7. Repeat the above steps until you get all the IMFs. The original signal can be expressed as

$$
x(t)=\sum_{k=1}^{K} c_{k}(t)+r_{K}(t)
$$

where $K$ represents the total number of IMFs.

\section{Sensitive Feature Extraction Based on Mutual Information}

3.1. Mutual Information. Mutual information can measure the degree of interdependence between the two variables, which means that the information content is shared between the two variables $[28,29]$. Given two random variables $X$ and $Y$, calculate their respective marginal probability distribution and joint probability distribution $p(x), p(y)$, and $p(x, y)$. The mutual information between them is

$$
\operatorname{MI}(x, y)=\sum_{x} \sum_{y} p(x, y) \log \frac{p(x, y)}{p(x) p(y)} .
$$

3.2. Sensitivity Coefficient Based on Mutual Information. The IMFs can be obtained by bivariate empirical mode decomposition (BEMD) for the fault vibration signals. However, not all the IMFs are important to the evaluation condition of machinery rotating, so it is significant to select the sensitive IMFs [30]. Firstly, orthogonal sampling technique is used to collect the vibration signals in the normal and fault state, which are recorded as $x_{z}(t)$ and $x(t)$. Then the fault vibration signal $x(t)$ is decomposed by BEMD. Get modal component IMFs, $c_{1}(t), \ldots, c_{n}(t)$.

(1) According to formula (6), the mutual information $\mathrm{MI}_{i}$ between the intrinsic mode components IMF $c_{i}(t)(i=$ $1,2, \ldots, n)$ and the fault vibration signals $x(t)$ is calculated. And do the normalization as follows:

$$
a_{i}=\frac{\mathrm{MI}_{i}}{\max \left(\mathrm{MI}_{i}\right)} .
$$

(2) Calculate the mutual information $\mathrm{MI}_{i}^{\prime}$ between the intrinsic mode components $\operatorname{IMF} c_{i}(t)(i=1,2, \ldots, n)$ and the normal vibration signals $x_{z}(t)$. The same normalization is done as follows:

$$
b_{i}=\frac{\mathrm{MI}_{i}^{\prime}}{\max \left(\mathrm{MI}_{i}^{\prime}\right)} \text {. }
$$

(3) Calculate the sensitivity coefficient of each modal component IMFs as follows:

$$
\lambda_{i}=\frac{a_{i}}{b_{i}}
$$

where $a_{i}$ represents the mutual information between the $n$th IMFs and their original signals and $b_{i}$ represents the mutual information between the $n$th IMFs and the normal signals. In order to make the component better reflect the characteristics of the original signal, $a_{i}$ should be as large as possible. In order to better monitor the characteristics of the fault signals, $b_{i}$ should be as small as possible. Considering the characteristics of the early fault signal of rotating machinery, the amplitude of the signal is smaller, and the mutual information between the IMFs and the original signal is relatively small. Therefore, this paper reflects the change of signal characteristic components by increasing the multiple relations. That is to say, the higher the $\lambda_{i}$ value is, the more sensitive the IMF is.

\section{Full Spectrum Theory}

There are usually two vertical sensors in the same section of the rotor for rotating machinery to extract vibration information. The full spectrum can fuse the information of vibration signals in two orthogonal directions and comprehensively express the intensity and spectrum structure of the rotor vibration $[31,32]$.

The core idea of the full spectrum is that rotating machinery shows whirling phenomena under the combination of harmonic frequencies. The whirling trajectory is a series of ellipses. The length of the long axis of the ellipse is defined as the principal vibration vector to evaluate vibration intensity. The length of the short axis of the ellipse is defined as the auxiliary vector to evaluate vibration intensity as auxiliary. And through the whirling intensity under the harmonic frequency of rotor, the rotating machinery fault is diagnosed and identified [33].

Set $X, Y$ direction of the data sequence as $\tilde{x}$ and $\tilde{y}$. They can constitute the complex sequence $\widetilde{z}=\widetilde{x}+i \widetilde{y}$. Carry on the Fourier transform for the complex sequence $\widetilde{z}$, and get the frequency domain complex signal $\widetilde{Z}$.

$$
\widetilde{Z}=\widetilde{X}+i \widetilde{Y}=\operatorname{Re}(Z)+\operatorname{Im}(Z) .
$$

The length of the long axis of the ellipse $R_{a i}$ is defined as the main vibration vector.

$$
R_{a i}=\frac{1}{2 N}\left[\left|Z_{i}\right|+\left|Z_{N-i}\right|\right] .
$$


The length of the short axis of the ellipse $R_{b i}$ is defined as vice vibration vector.

$$
R_{b i}=\frac{1}{2 N}\left[\left|Z_{i}\right|-\left|Z_{N-i}\right|\right]
$$

and $\alpha_{i}$ is the angle between the main vibration vector and the $X$ axis.

$$
\tan 2 \alpha_{i}=\frac{\operatorname{Im} Z_{i} \operatorname{Re} Z_{N-i}-\operatorname{Re} Z_{i} \operatorname{Im} Z_{N-i}}{\operatorname{Re} Z_{i} \operatorname{Im} Z_{N-i}-\operatorname{Im} Z_{i} \operatorname{Re} Z_{N-i}}
$$

$\phi_{i}$ is the initial phase angle of the elliptic trajectory at this frequency, whose calculation formula can be obtained according to the property of Fourier transform (the details of derivation were shown in [24]):

$$
\tan \phi_{i}=\frac{\operatorname{Im} Z_{i}+\operatorname{Im} Z_{N-i}}{\operatorname{Re} Z_{i}+\operatorname{Re} Z_{N-i}}
$$

where $i=1,2, \ldots, N / 2-1$.

Therefore, utilize Fourier transform for the vibration signals in two orthogonal directions and obtain the characteristic information of each direction, whose full spectrum is required, which can simplify the calculation between complex Fourier parameters. This method not only greatly reduces the amount of calculation, but also is very accurate and established a contact with the conventional analysis method. When the information source is a single source, the algorithm is still established, fully meeting the requirements of real-time detection and analysis.

\section{Coupling Fault Feature Extraction Method Based on BEMD and Full Spectrum}

Owing to the complexity, coupling, and uncertainty of rotating machinery faults, the intrinsic dynamic characteristics of the faults are more complicated. The external manifestation is that the vibration signals in different directions may indicate different characteristic information, and the singlechannel signal characteristics diagnosis for rotary machinery is prone to misjudgment and leakage judgment. And BEMD and full spectrum have advantages in the processing of two-dimensional signals and information fusion. Therefore, this paper proposes a new coupling fault feature extraction method based on BEMD and full spectrum for rotating machinery. To select the sensitive IMF, use the sensitivity coefficient based on mutual information as the screening criteria. The extraction process of this method is shown in Figure 1. Specific steps are as follows.

Step 1. Collect the orthogonal vibration signal of the rotating machine in the normal and fault condition by the sensor.

Step 2. The orthogonal fault vibration signal is decomposed by BEMD to obtain $K$ intrinsic mode components $c_{k}(k=$ $1,2, \ldots, K)$.

Step 3. Calculate the sensitivity of each IMF.

Step 4. Select the modal component IMFs with the larger sensitive coefficient as sensitive modal components IMFs.

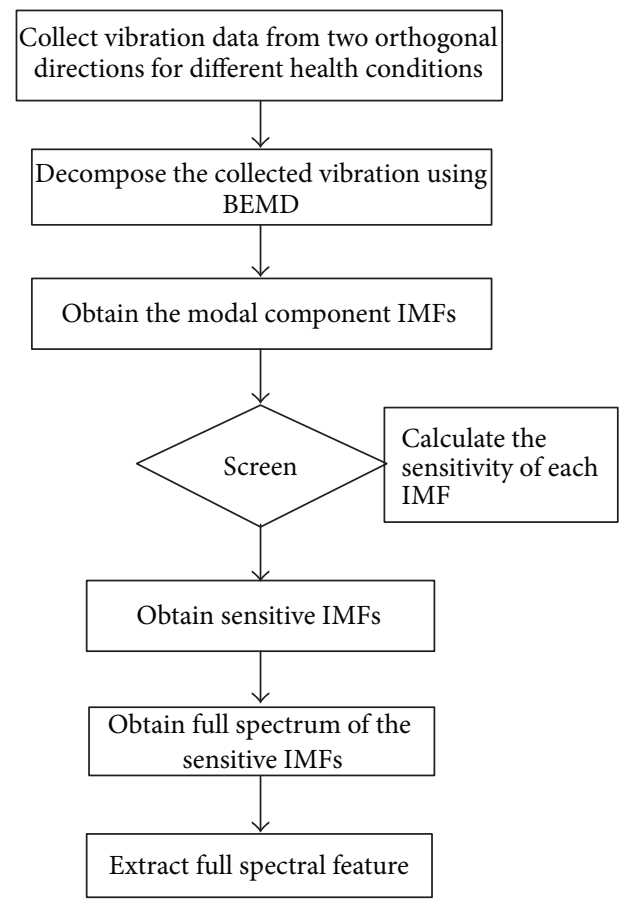

FIGURE 1: Flow chart of the proposed method.

Step 5. Full spectrum analysis of the selected modal components IMFs is carried out to detect the characteristic frequency of coupling fault signals.

\section{Experiment Results}

6.1. Simulation Experiment. There are two common phenomena, a change in amplitude at the typical frequency [34] and a new frequency appearance [35], when rotating machinery breaks down. In this paper, the vibration signal of the hydropower unit bearing is simulated. Suppose that the rotating frequency of hydropower unit is $f_{0}$, It is considered that the vibration fault signal of the actual hydroelectric generating set often contains $f_{0}, 2 f_{0}, 3 f_{0}, 50 \mathrm{~Hz}, 100 \mathrm{~Hz}$, and some common characteristic signals and noise. In order to make the simulation result more fitting with the actual situation and verify the effectiveness of the proposed method, this paper, in the processing of two-dimensional signals, simulates the normal and fault signals of the hydropower unit. The specific simulation vibration signal is as follows:

$$
\begin{aligned}
x_{z}= & 8 \sin (\omega t)+5 \sin (5 \omega t)+3 \sin (100 \pi t) \\
& +2 \operatorname{rand}(1, N) \\
y_{z}= & 8 \cos (\omega t)+5 \cos (5 \omega t)+3 \cos (100 \pi t) \\
& +2 \operatorname{rand}(1, N) \\
x= & 8 \sin (\omega t)+5 \sin (5 \omega t)+5 \sin (100 \pi t) \\
& +2 \operatorname{rand}(1, N)+5 \sin (2 \omega t)
\end{aligned}
$$


TABLE 2: Sensitivity coefficient of each characteristic component.

\begin{tabular}{lccccc}
\hline IMF & IMF1 & IMF2 & IMF3 & IMF4 & IMF5 \\
\hline Sensitivity coefficient & $\mathbf{2 . 8 8 1}$ & 0.693 & $\mathbf{1 . 0 7 8}$ & 0.767 & 0.654 \\
\hline
\end{tabular}

$$
\begin{aligned}
y= & 8 \cos (\omega t)+5 \cos (5 \omega t)+5 \cos (100 \pi t) \\
& +2 \operatorname{rand}(1, N)+5 \cos (2 \omega t),
\end{aligned}
$$

where the $x_{z}$ and $y_{z}$ are used to simulate the normal signals, which are composed of a rotating component at a frequency of $f_{0}$ with amplitude of 8 , a rotating component at a frequency of $5 f_{0}$ with amplitude of 5 , a rotating component at a frequency of $50 \mathrm{~Hz}$ with amplitude of 3 , and the noise generated randomly with amplitude of 2 . Each component of the normal vibration signal is shown in Figure 2(a). Figure 2(a) separately represents the rotating component at a frequency of $f_{0}, 5 f_{0}$, $50 \mathrm{~Hz}$, and the noise generated randomly in turn.

The $x$ and $y$ are used to simulate the fault signals, which not only are composed components of the normal signal, but also have the rotating component at a frequency of $2 f_{0}$ with amplitude of 5 and the rotating component at a frequency of $50 \mathrm{~Hz}$ with increased amplitude of 2 . Each component of the fault vibration signal is shown in Figure 2(b). And Figure 2(b) separately represents the rotating component at a frequency of $f_{0}, 5 f_{0}, 50 \mathrm{~Hz}$, the noise generated randomly, and the rotating component at a frequency of $2 f_{0}$ in turn, where $\omega$ represents the angular velocity, in actual hydroelectric generating set, the rated speed is $107.1 \mathrm{r} / \mathrm{min}$, the sampling frequency is $227 \mathrm{~Hz}$, and the sampling point is 1024. Considering the consistency with the actual operation of hydropower units and the simplicity of simulation, it can be assumed that the unit speed is $107 \mathrm{r} / \mathrm{min}$, sampling frequency is $250 \mathrm{~Hz}$, and sampling point $N$ is 1000 . The normal and the fault raw signal are shown in Figure 3, which separately represent the normal signal of the $X$ direction, the normal signal of the $Y$ direction, the fault signal of the $X$ direction, and the fault signal of the $Y$ direction in turn.

The proposed method is used to extract the feature of the simulation signal in Figure 3. Firstly, the orthogonal fault raw signal is decomposed by BEMD, and the results are shown in Figure 4 . Where the blue curve represents the real part and the black one represents the imaginary part of the decomposition results by BEMD. Then calculate the sensitivity coefficient of each IMF, as shown in Table 2.

The IMF1 and IMF3, whose sensitivity coefficient is greater than 1, are selected as sensitive IMFs. Utilize the full vector envelope technique to fuse the sensitive IMFs selected and obtain full spectrum of the sensitive IMFs as shown in Figure 5.

Based on the full spectrum of the sensitive IMFs in Figure 5, the method proposed in the paper can fuse the orthogonal signals in two directions. And it can accurately detect the new frequency appearance at a frequency of $5 f_{0}$ and the change in amplitude at the typical frequency of $50 \mathrm{~Hz}$. Obviously, the method has strong compatibility and effectiveness.
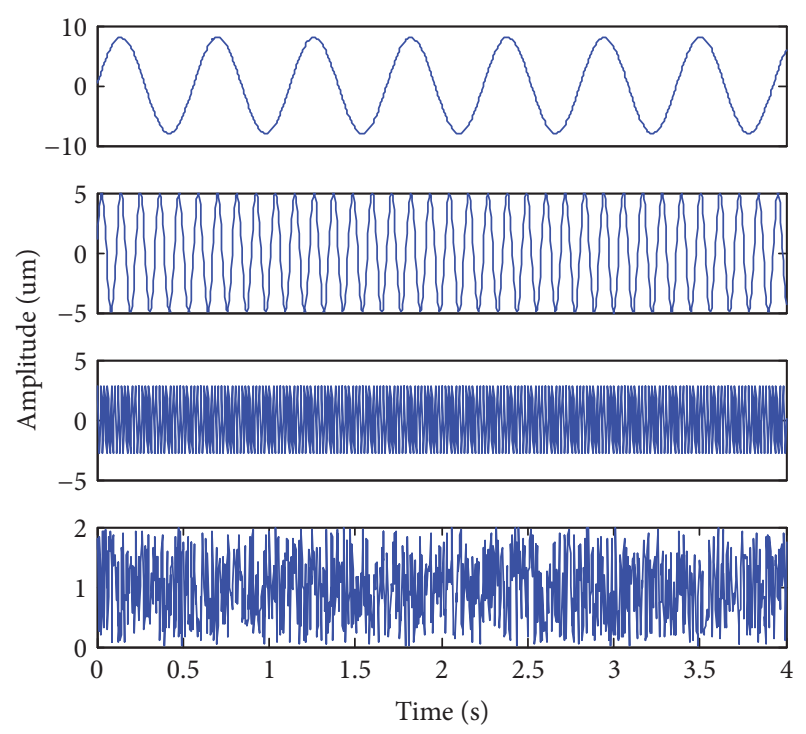

(a) Components of normal vibration signal

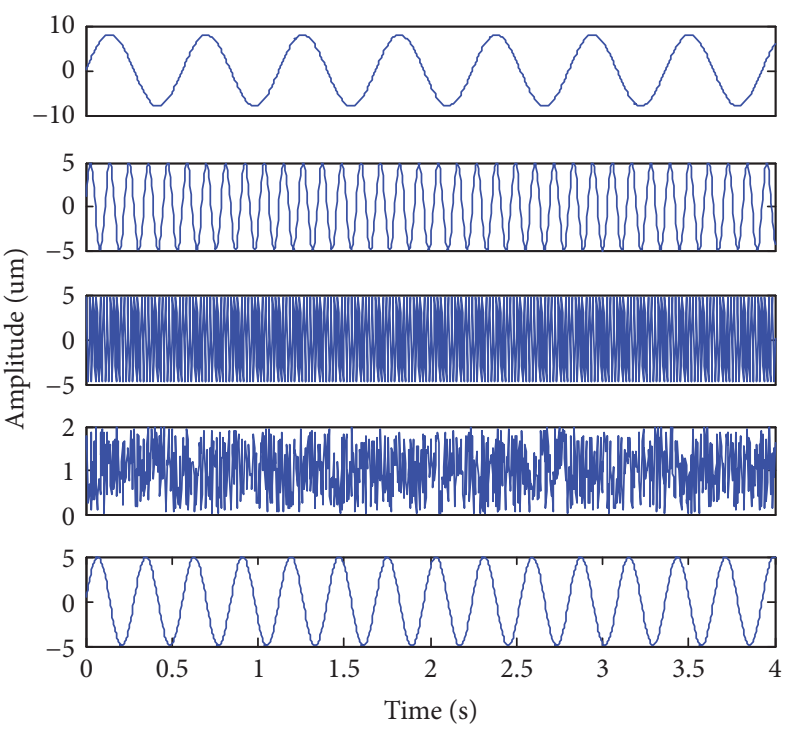

(b) Components of fault vibration signal

FIGURE 2: Components of normal and fault vibration signal.

6.2. Coupling Fault Feature Extraction of Turbine Guide Bearing. To verify the effectiveness of the proposed method in coupling fault feature extraction for rotating machinery, the experimental studies on a hydroelectric turbine in upper reaches of Yellow River are conducted. The experimental signals are acquired from the prototype of hydroelectric turbine with 5 blades and 16 guide vanes, the maximum water head is $25.7 \mathrm{~m}$, the rated head is $16 \mathrm{~m}$, the rated power of the turbine is about $49 \mathrm{MW}$, and the rated speed is $107.1 \mathrm{r} / \mathrm{min}(1.79 \mathrm{~Hz})$. Figure 6 describes specific layout of measuring points of pressure fluctuation signals in the turbine [36].

The measured data are collected turbine guide bearing by the vibration and throw monitoring sensors turbine guide bearing (As shown in Figure 6), which are from horizontal and vertical direction. Randomly select a segment of signal 

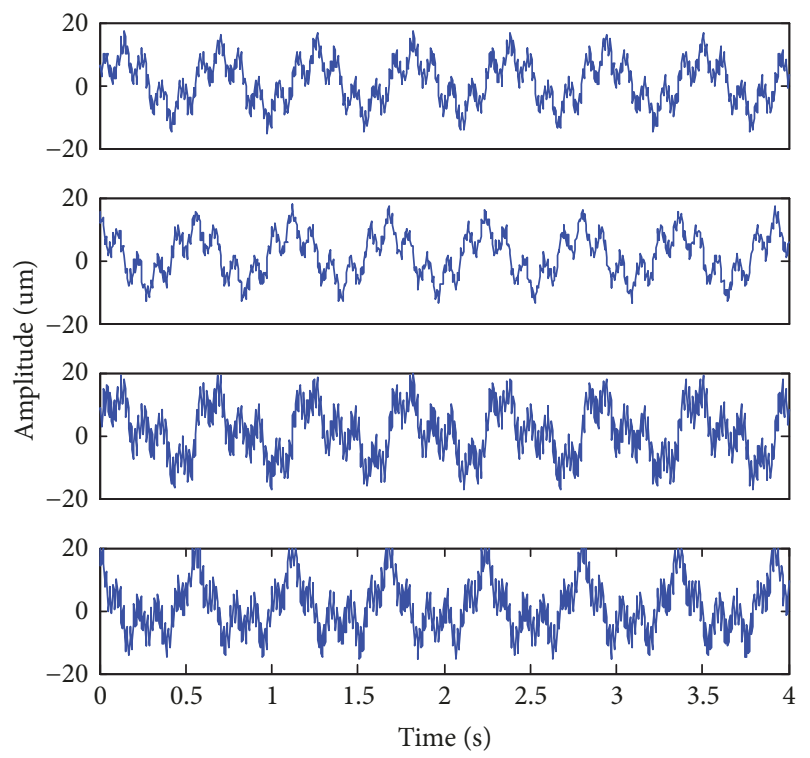

FIGURE 3: Observation signal of normal and faulty.

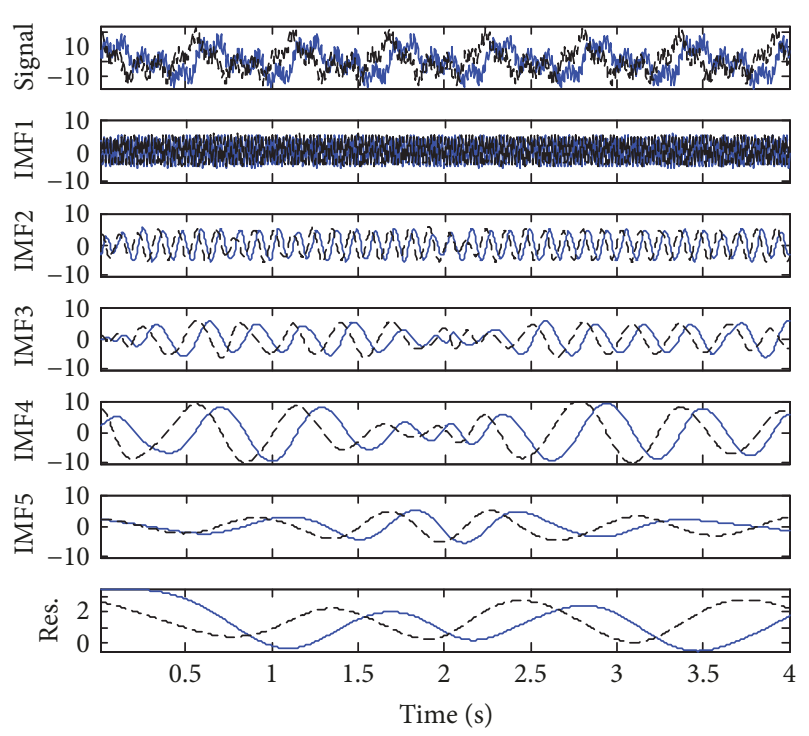

FIGURE 4: Results of BEMD decomposition for fault signal.

from normal and fault condition to verify the effectiveness of the proposed method, as shown in Figure 7: from top to bottom, the normal signal of the $X$ direction, the normal signal of the $Y$ direction, the fault signal of the $X$ direction, and the fault signal of the $Y$ direction, where the sampling frequency is $227 \mathrm{~Hz}$ and the sampling point $M$ is 1024 . Under the same load, the fault raw data is collected when the unit is abnormal.

Using the method proposed in this paper to extract the coupling fault feature of turbine guide bearing, firstly, the fault signal is decomposed by BEMD, and the results of the decomposition are shown in Figure 8 (where the blue represents the real part and the black represents the imaginary part). As shown in Figure 8, the BEMD decomposition

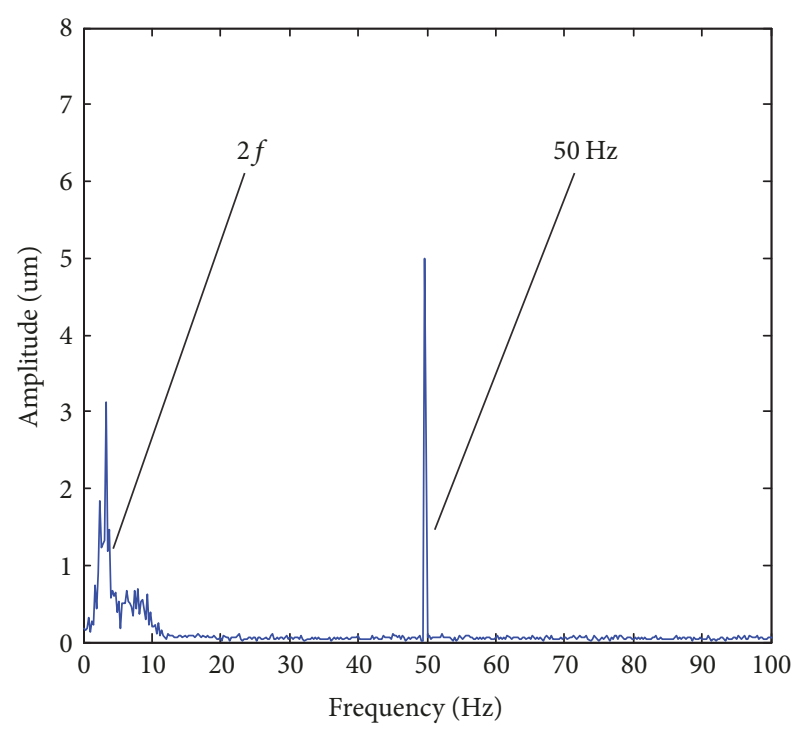

FIGURE 5: Full spectrum of sensitive IMFs.

can effectively preserve the phase information of the orthogonal two-dimensional signal, and the decomposition results have good synchronization. In order to illustrate the advantages of BEMD, the real and imaginary parts of the fault signal are separately decomposed by EMD, and the results are shown in Figure 9. Obviously, when utilizing EMD to decompose the orthogonal two-dimensional signal, the phase information contained in the orthogonal signal is lost. In addition, compared to the decomposition results of BEMD, the decomposition component of EMD is too much, that is, the phenomenon of overdecomposition.

On the basis of BEMD decomposition, the sensitivity coefficients of each IMFs are calculated, as shown in Table 3. And select the sensitive coefficient of large IMF2, IMF3, and IMF5 as the sensitive component IMFs. Then utilize the full vector envelope technique to fuse the sensitive IMFs selected, and obtain the full spectrum of the sensitive IMFs as shown in Figure 10. Obtain the decomposition results of EMD to compare them with the decomposition results of BEMD as shown in Figure 11. Calculate the sensitivity coefficients of the IMFs obtained by EMD in two directions, as shown in Table 4 . In the IMFs of the real part, IMF2, IMF3, and IMF6 with the larger sensitivity coefficient are selected as the sensitive IMFs, and In the IMFs of the imaginary part, IMF2, IMF4, and IMF5 with the larger sensitivity coefficient are selected as the sensitive IMFs. The frequency spectrum analysis of the sensitivity coefficient in each direction is carried out. The frequency spectrum of the EMD sensitive IMFs is shown in Figure 11 (where the upper part represents the real part and the lower part represents the imaginary part of the decomposition results by BEMD).

It can be seen from Figure 11 that the vibration signal (real part) in the horizontal direction can be divided into $2 f_{0}$, a small number of the 3 -frequency $3 f_{0}$ and $15 f_{0}$. However, the vibration signal in the vertical direction (imaginary part) contains $1 f_{0}, 15 f_{0}$, and $50 \mathrm{~Hz}$ vibration signal. Therefore, the extraction results for vibration signals in the two orthogonal 


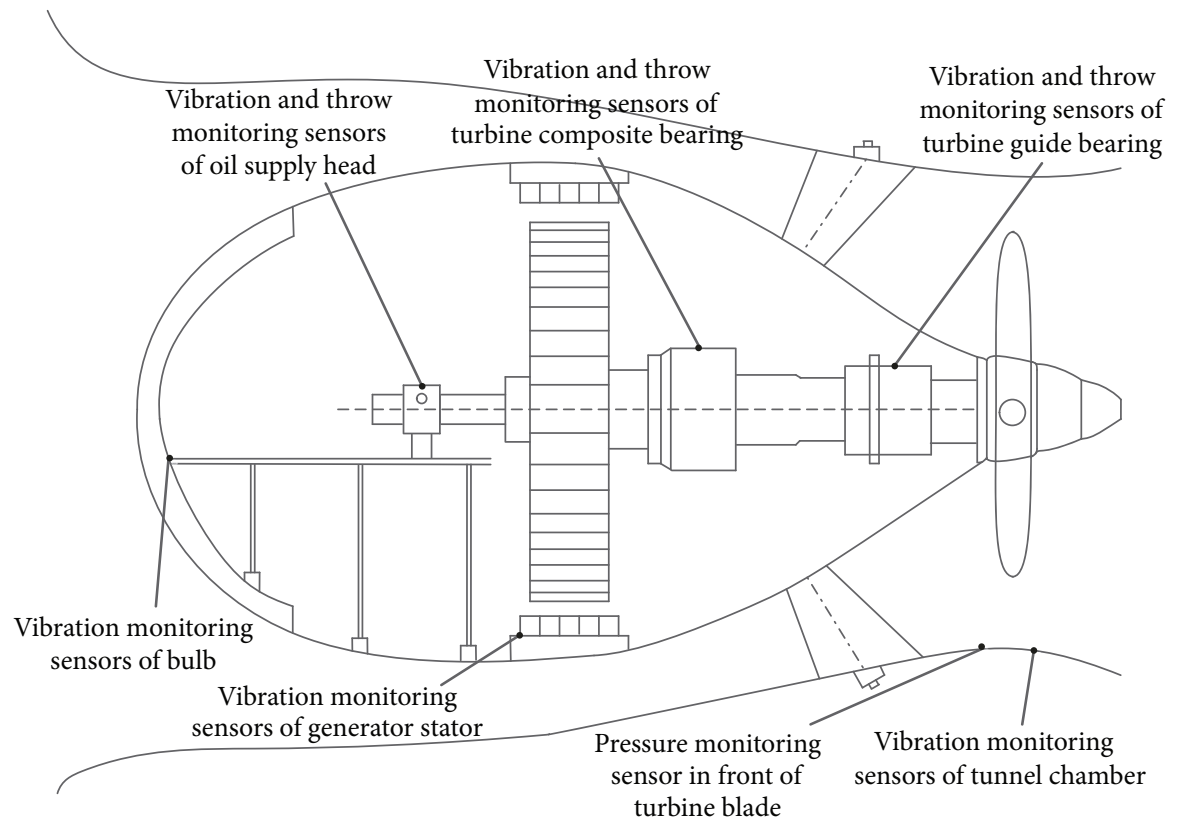

FIGURE 6: Specific layout of measuring point in hydroelectric turbine.
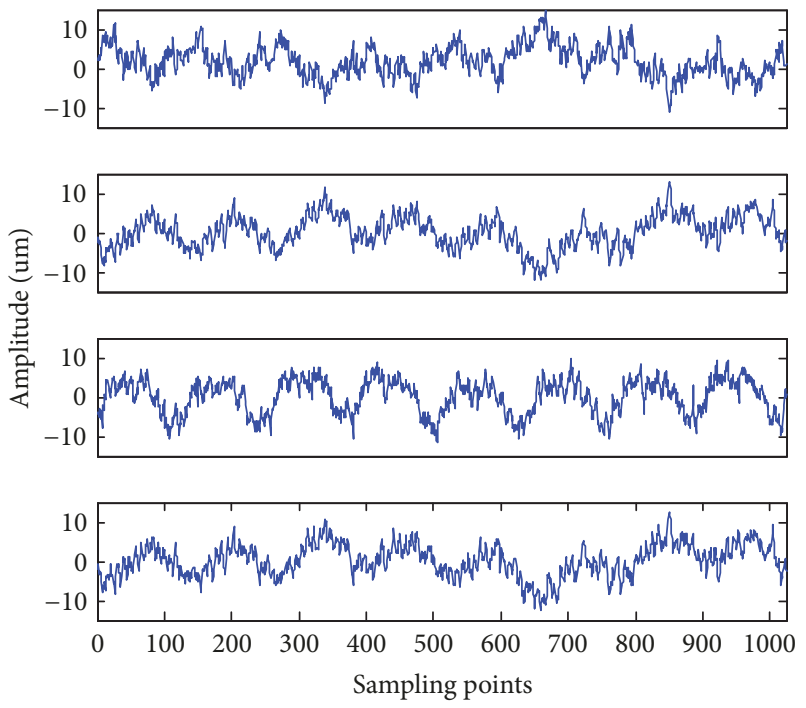

FIGURE 7: Raw vibration data of normal and fault signals.

directions are different due to the certain interference, which often brings trouble for condition monitoring of rotating machinery. Compared with this, the method proposed in this paper can solve the problem of the inconsistency between the two channels. As shown in Figure 10, this method can better fuse the information in two orthogonal directions of vibration signal. This method has good compatibility, which can be more comprehensive and accurate to detect the vibration signal in the rotating machinery, and the feature extraction results are more reliable.

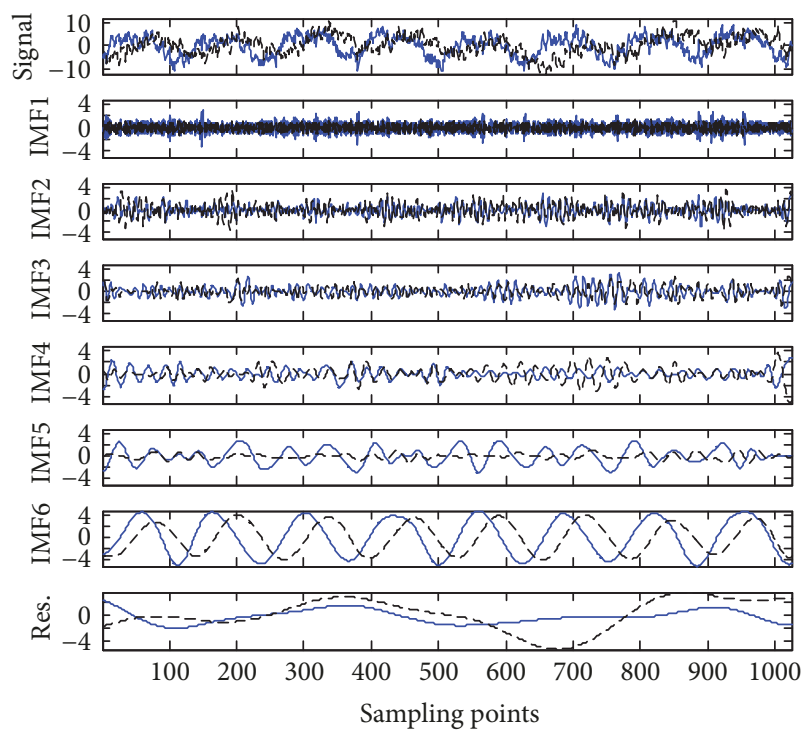

Figure 8: BEMD decomposition results of measured fault signal.

\section{Conclusions}

This paper proposed a method to extract a feature from orthogonal signals in two-direction sensors for the condition monitoring of rotating machinery based on bivariate empirical mode decomposition (BEMD) and full spectrum.

BEMD is employed to decompose signals from two orthogonal sources together; thus a complicated rotation can be represented by a set of simpler rotation components. BEMD is proved to outperform standard EMD for two orthogonal 


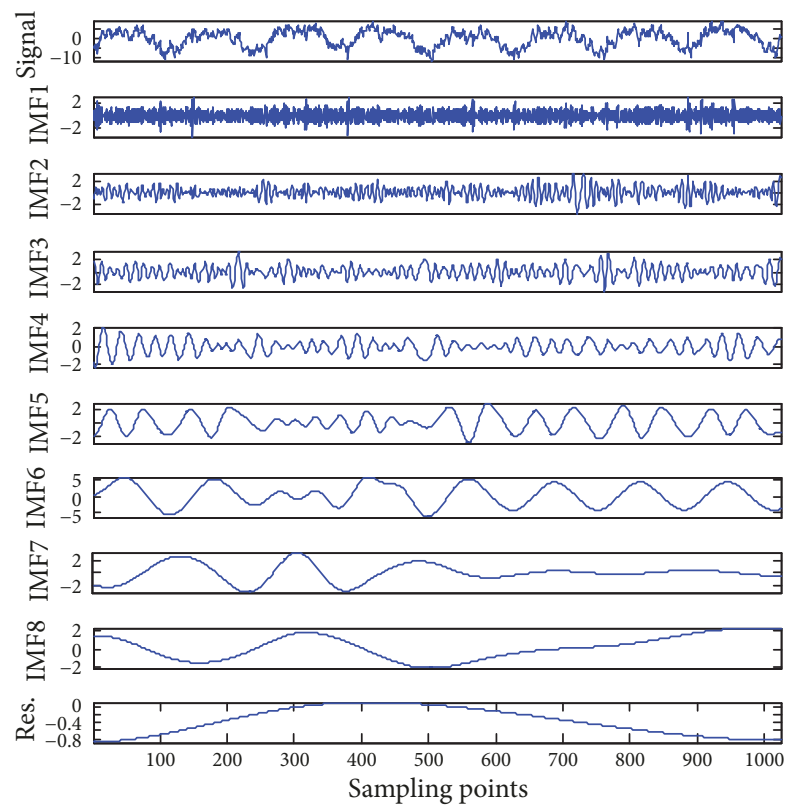

(a) The decomposition results of real fault signal

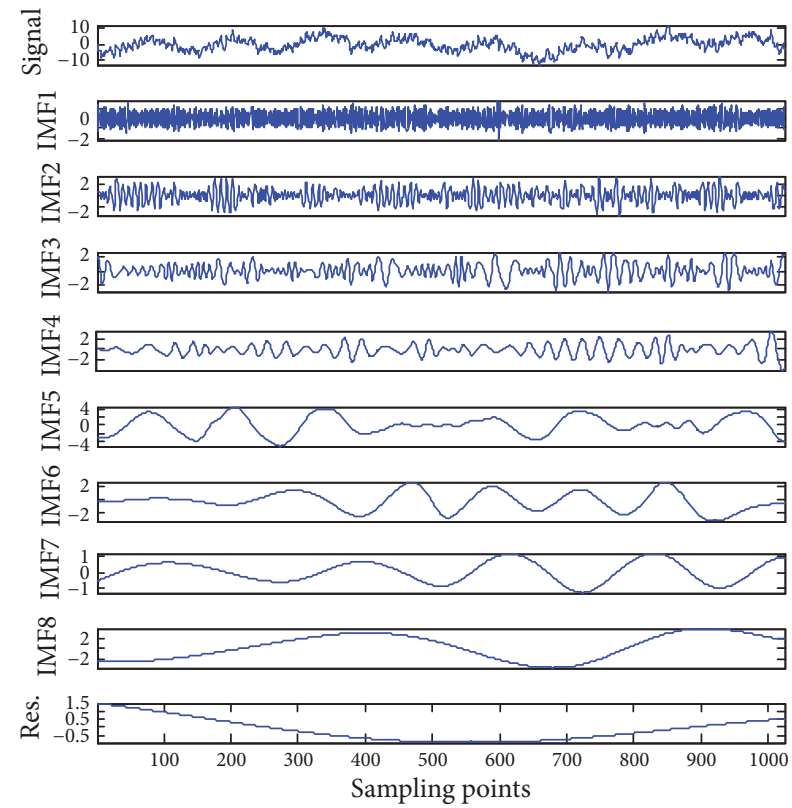

(b) The decomposition results of imaginary part fault signal

FIGURE 9: EMD decomposition results of real and imaginary part of measured fault signal.

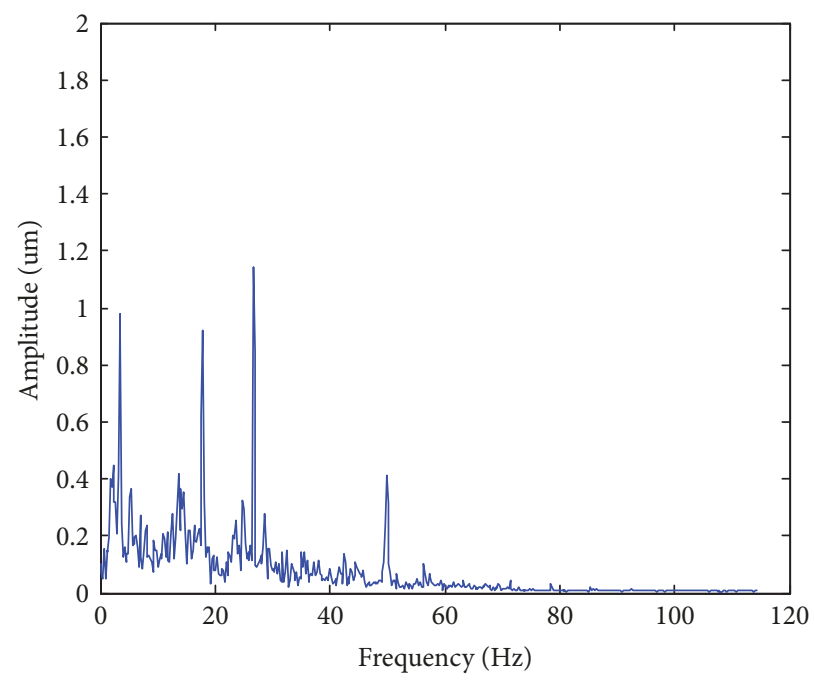

FIGURE 10: Full vector envelope spectrum of BEMD.

TABLE 3: Sensitivity coefficients of BEMD modal components.

\begin{tabular}{llllll}
\hline IMFs & IMF1 & IMF2 & IMF3 & IMF4 & IMF5 \\
\hline Sensitivity coefficient & 0.661 & $\mathbf{1 . 0 4 3}$ & $\mathbf{1 . 1 7 2}$ & 0.564 & $\mathbf{0 . 9 8 4}$ \\
\hline
\end{tabular}

TABLE 4: Sensitivity coefficients of EMD modal components.

\begin{tabular}{lccccccccc}
\hline IMFs & IMF1 & IMF2 & IMF3 & IMF4 & IMF5 & IMF6 & IMF7 & IMF8 & IMF9 \\
\hline Real part & 0.831 & $\mathbf{1 . 6 3 4}$ & $\mathbf{1 . 8 7 5}$ & 0.73 & 0.455 & $\mathbf{1 . 3 4 4}$ & 0.495 & 0.393 & 0.543 \\
Imaginary part & 0.76 & $\mathbf{2 . 2 9 2}$ & 0.877 & $\mathbf{1 . 1 2}$ & $\mathbf{1 . 8 5 3}$ & 0.742 & 0.436 & 0.874 & 0.432 \\
\hline
\end{tabular}



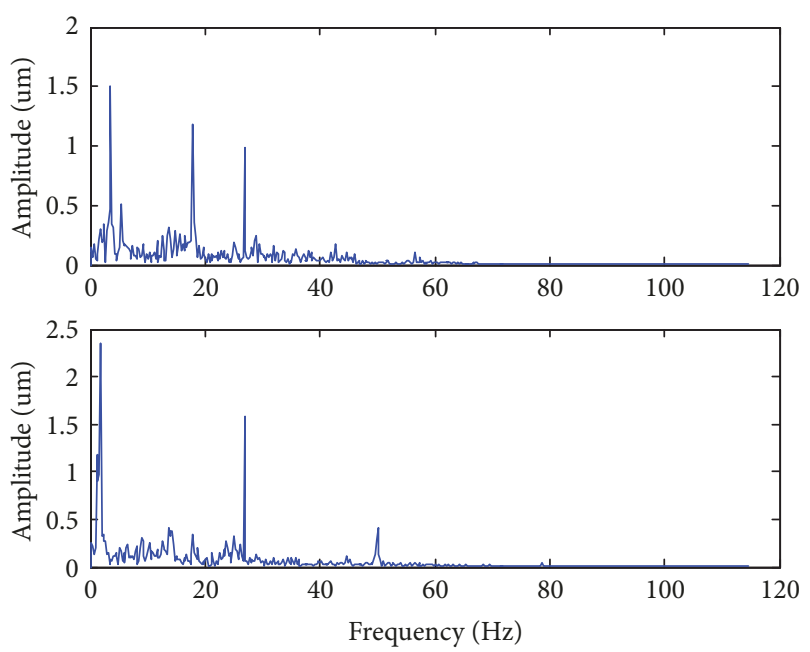

FIGURE 11: Spectrum of EMD decomposition sensitive component.

signals, because the intrinsic mode functions (IMFs) derived by BEMD preserve the signal phase information, detect synchronous features contained in the two-dimensional signal, and effectively solve the problem of misjudgment and leakage judgment in extract feature method by single-channel signal for rotary machinery. Thus the comparison of IMFs for different health conditions is made reasonable and easy.

A criterion based on mutual information is proposed for selecting the most sensitive IMF. The definition of this standard is to better represent the IMF of the original signal and penalize IMFs that cannot distinguish between different health conditions. Therefore, the IMF selected always ensures that it retains unique information about a particular health condition.

The performance of the proposed method has been evaluated for the hydroelectric turbine in upper reaches of Yellow River. Experiments results verified the effectiveness of the proposed method in two orthogonal directions, fault feature extraction and fault diagnosis for rotating machinery.

\section{Conflicts of Interest}

The authors declare that they have no conflicts of interest.

\section{Acknowledgments}

This work was supported by the National Natural Science Foundation of China (nos. 51779206, 51279161) and the science and technology projects of Water Resources Department of Shaanxi Province (no. 2015slkj-04). Furthermore, the authors are grateful to the staff of China Electric Power Research Institute and State Grid Gansu Province Electric Power Research Institute.

\section{References}

[1] X.-H. He, D. Wang, Y.-F. Li, and C.-H. Zhou, "A novel bearing fault diagnosis method based on gaussian restricted boltzmann machine," Mathematical Problems in Engineering, vol. 2016, Article ID 2957083, 8 pages, 2016.

[2] X. Li, A. Zheng, X. Zhang, C. Li, and L. Zhang, "Rolling element bearing fault detection using support vector machine with improved ant colony optimization," Measurement, vol. 46, no. 8, pp. 2726-2734, 2013.

[3] G. Lang, J. Lin, Y. Liao, and M. Zhao, "Impact of system anisotropy on vibration reduction of rotating machinery and its evaluation method," Mechanical Systems and Signal Processing, vol. 93, pp. 299-311, 2017.

[4] L. Liang, F. Liu, M. Li, and G. Xu, "Manifold learning with selforganizing mapping for feature extraction of nonlinear faults in rotating machinery," Mathematical Problems in Engineering, vol. 2015, Article ID 873905, 11 pages, 2015.

[5] A. Cohen, Numerical Analysis of Wavelet Methods, Elsevier, 2003.

[6] Y. Feng, J.-R. Cao, B.-C. Lu, and D.-F. Zhang, "Fault feature extraction method for rolling bearing based on wavelet transform optimized by continuous kurtosis," Zhendong yu Chongji, vol. 34, no. 14, pp. 27-32, 2015.

[7] N. E. Huang, Z. Shen, S. R. Long et al., "The empirical mode decomposition and the Hilbert spectrum for nonlinear and nonstationary time series analysis," Proceedings A, vol. 454, pp. 903995, 1998.

[8] C. Junsheng, Y. Dejie, and Y. Yu, "A fault diagnosis approach for roller bearings based on EMD method and AR model," Mechanical Systems and Signal Processing, vol. 20, no. 2, pp. 350-362, 2006.

[9] K. Dragomiretskiy and D. Zosso, "Variational mode decomposition," IEEE Transactions on Signal Processing, vol. 62, no. 3, pp. 531-544, 2014.

[10] S. Liu, G. Tang, X. Wang, and Y. He, "Time-frequency analysis based on improved variational mode decomposition and teager energy operator for rotor system fault diagnosis," Mathematical Problems in Engineering, vol. 2016, Article ID 1713046, 9 pages, 2016.

[11] H. Keskes, A. Braham, and Z. Lachiri, "Broken rotor bar diagnosis in induction machines through stationary wavelet packet transform and multiclass wavelet SVM," Electric Power Systems Research, vol. 97, pp. 151-157, 2013.

[12] D. B. Percival and A. T. Walden, Wavelet Methods for Time Series Analysis, Cambridge University Press, Cambridge, UK, 2006.

[13] L. Saidi, J. B. Ali, and F. Fnaiech, "Bi-spectrum based-EMD applied to the non-stationary vibration signals for bearing faults diagnosis," ISA Transactions, vol. 53, no. 5, pp. 1650-1660, 2014.

[14] J. Cheng, D. Yu, and Y. Yang, "A fault diagnosis approach for gears based on IMF AR model and SVM," EURASIP Journal on Advances in Signal Processing, vol. 2008, Article ID 647135, 2008.

[15] Z. Li, J. Chen, Y. Zi, and J. Pan, "Independence-oriented VMD to identify fault feature for wheel set bearing fault diagnosis of high speed locomotive," Mechanical Systems and Signal Processing, vol. 85, pp. 512-529, 2017.

[16] L. Hou, Y. Chen, Q. Cao, and Z. Lu, "Nonlinear vibration analysis of a cracked rotor-ball bearing system during flight maneuvers," Mechanism and Machine Theory, vol. 105, pp. 515-528, 2016.

[17] D. P. Mandic, S. L. Goh, and K. Aihara, "Sequential data fusion via vector spaces: fusion of heterogeneous data in the complex domain," Journal of Signal Processing Systems, vol. 48, no. 1-2, pp. 99-108, 2006. 
[18] P. Goldman and A. Muszynska, "Application of full spectrum to rotating machinery diagnostics, Orbit," First Quarter, pp. 17-21, 1999.

[19] G. Rilling, P. Flandrin, P. Goncalves, and J. M. Lilly, "Bivariate empirical mode decomposition," IEEE Signal Processing Letters, vol. 14, no. 12, pp. 936-939, 2007.

[20] D. Looney and D. P. Mandic, "Multiscale image fusion using complex extensions of EMD," IEEE Transactions on Signal Processing, vol. 57, no. 4, pp. 1626-1630, 2009.

[21] I. Tsoumas, G. Georgoulas, A. Safacas, and G. Vachtsevanos, "Empirical mode decomposition of the stator start-up current for rotor fault diagnosis in asynchronous machines," in Proceedings of the 2008 International Conference on Electrical Machines, ICEM'08, 6, 1 pages, Vilamoura, Portugal, September 2008.

[22] M. K. I. Molla, P. R. Ghosh, and K. Hirose, "Bivariate EMDbased data adaptive approach to the analysis of climate variability," Discrete Dynamics in Nature and Society, vol. 2011, Article ID 935034, 21 pages, 2011.

[23] W. X. Yang, R. Court, P. J. Tavner, and C. J. Crabtree, "Bivariate empirical mode decomposition and its contribution to wind turbine condition monitoring," Journal of Sound and Vibration, vol. 330, no. 15, pp. 3766-3782, 2011.

[24] T. H. Patel and A. K. Darpe, "Use of full spectrum cascade for rotor rub identification," Advances in Vibration Engineering, vol. 8, no. 2, pp. 139-151, 2009.

[25] A. Okatan, C. Hajiyev, and U. Hajiyeva, "Fault detection in sensor information fusion Kalman filter," AEÜ - International Journal of Electronics and Communications, vol. 63, no. 9, pp. 762768, 2009.

[26] X. Liu, L. Ma, and J. Mathew, "Machinery fault diagnosis based on fuzzy measure and fuzzy integral data fusion techniques," Mechanical Systems and Signal Processing, vol. 23, no. 3, pp. 690700, 2009.

[27] K. Elbhbah and J. K. Sinha, "Vibration-based condition monitoring of rotating machines using a machine composite spectrum," Journal of Sound and Vibration, vol. 332, no. 11, pp. 28312845, 2013.

[28] X. J. Wang, Y. P. Cai, and X. Z. Lin, "ICE fault diagnosis method based on mutual information and WVD time-frequency analysis," Applied Mechanics and Materials, vol. 525, pp. 741-745, 2014.

[29] F. Li, J. Wang, M. K. Chyu, and B. Tang, "Weak fault diagnosis of rotating machinery based on feature reduction with Supervised Orthogonal Local Fisher Discriminant Analysis," Neurocomputing, vol. 168, pp. 505-519, 2015.

[30] H. Shao, H. Jiang, F. Wang, and H. Zhao, "An enhancement deep feature fusion method for rotating machinery fault diagnosis," Knowledge-Based Systems, vol. 119, pp. 200-220, 2017.

[31] N. Lu, Z. Xiao, and O. P. Malik, "Feature extraction using adaptive multiwavelets and synthetic detection index for rotor fault diagnosis of rotating machinery," Mechanical Systems and Signal Processing, vol. 52-53, no. 1, pp. 393-415, 2015.

[32] X. Zhao, T. H. Patel, and M. J. Zuo, "Multivariate EMD and full spectrum based condition monitoring for rotating machinery," Mechanical Systems and Signal Processing, vol. 27, no. 1, pp. 712728, 2012.

[33] P. Goldman, "A Muszynska . Application of full spectrum to rotating machinery diagnostics," Orbit, pp. 17-21, 1999.

[34] H.-Q. Wang, W. Hou, G. Tang, H.-F. Yuan, Q.-L. Zhao, and $\mathrm{X}$. Cao, "Fault detection enhancement in rolling element bearings via peak-based multiscale decomposition and envelope demodulation," Mathematical Problems in Engineering, vol. 2014, Article ID 329458, 11 pages, 2014.
[35] F. Chu and W. Lu, "Experimental observation of nonlinear vibrations in a rub-impact rotor system," Journal of Sound and Vibration, vol. 283, no. 3-5, pp. 621-643, 2005.

[36] J. Dang, R. Jia, X. Luo, H. Wu, and D. Chen, "Partly duffing oscillator stochastic resonance method and its application on mechanical fault diagnosis," Shock and Vibration, vol. 2016, Article ID 3109385, 14 pages, 2016. 


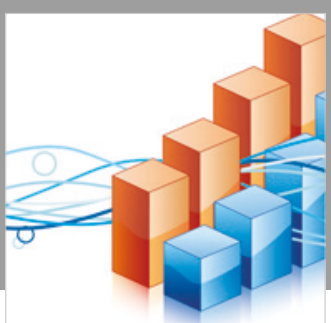

Advances in

Operations Research

\section{-n-m}
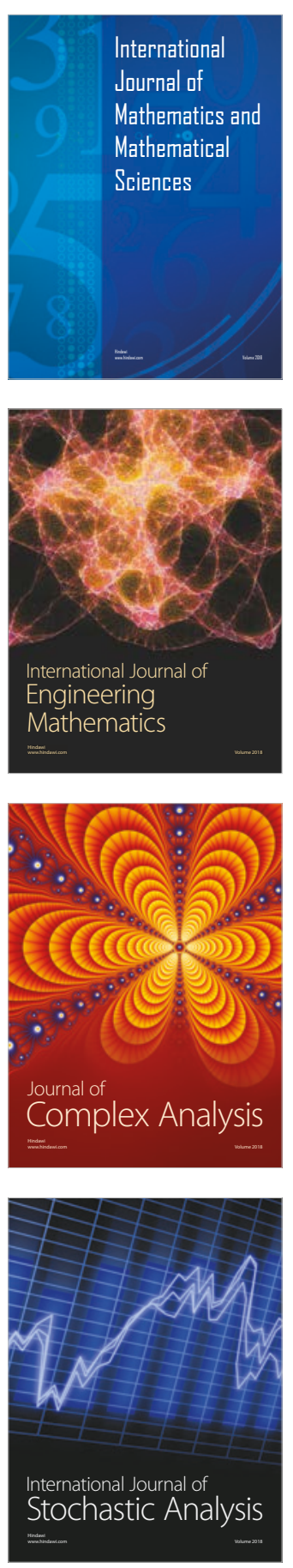
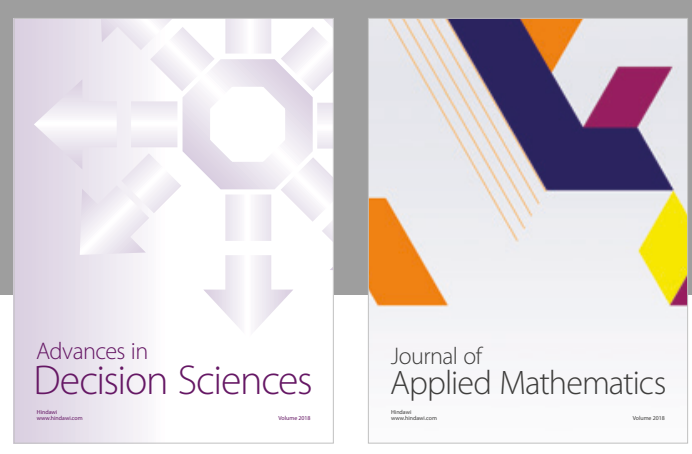

Journal of

Applied Mathematics
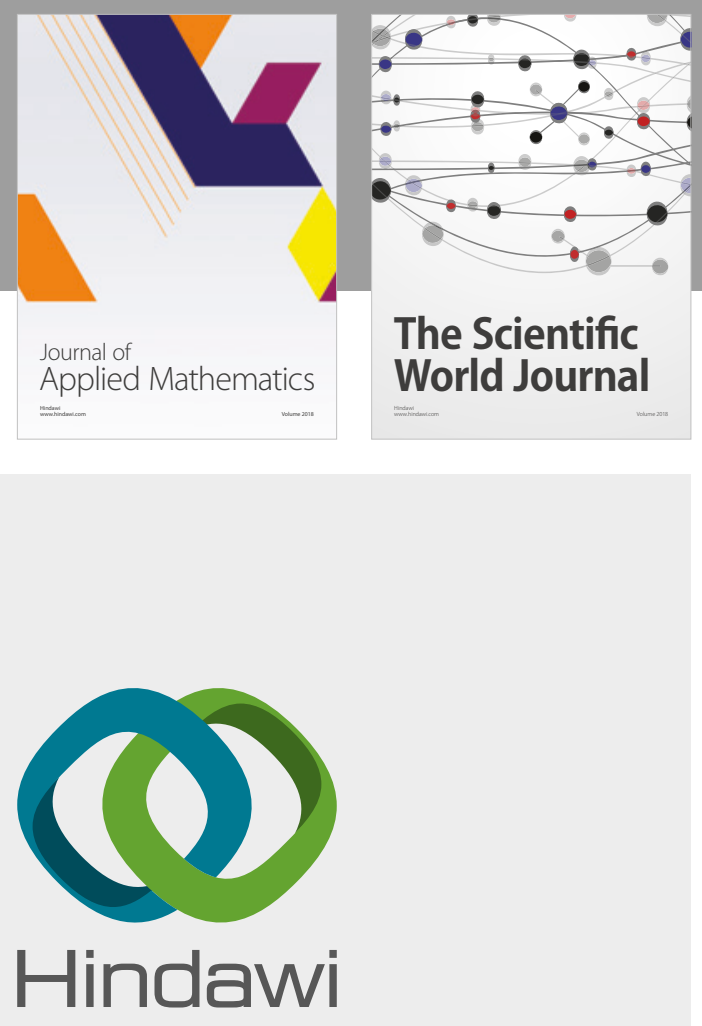

Submit your manuscripts at

www.hindawi.com

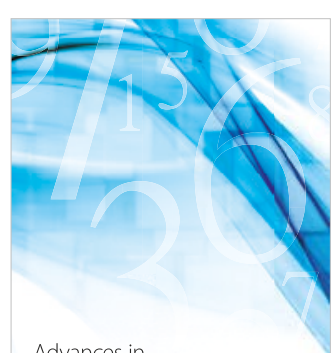

Advances in
Numerical Analysis
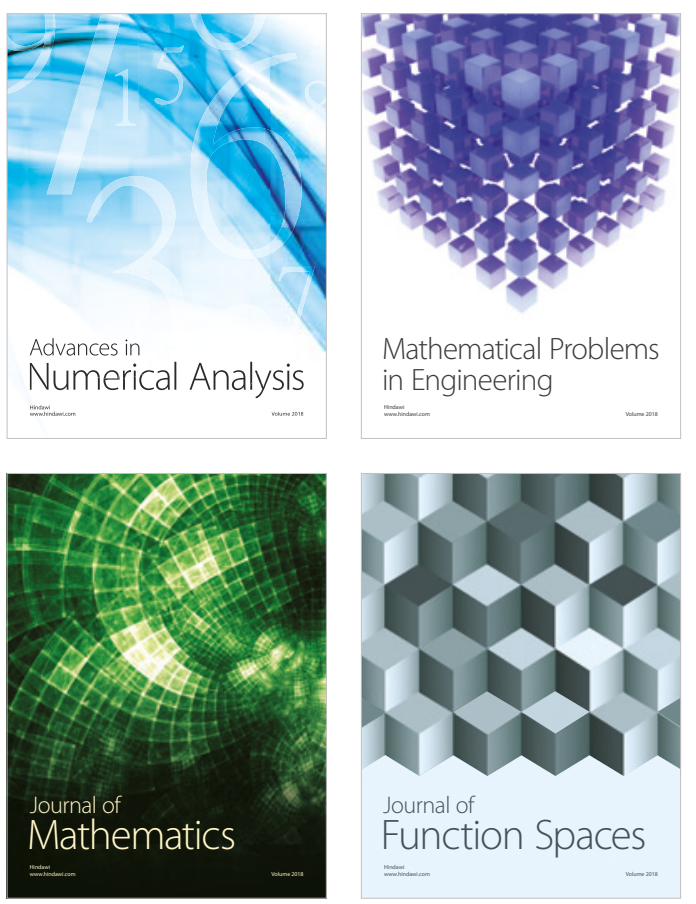

Mathematical Problems in Engineering

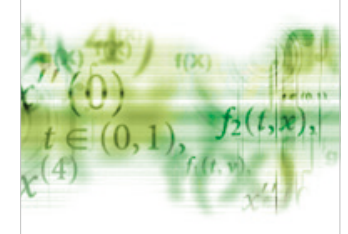

International Journal of

Differential Equations

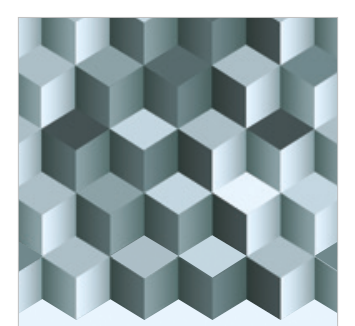

Journal of

Function Spaces

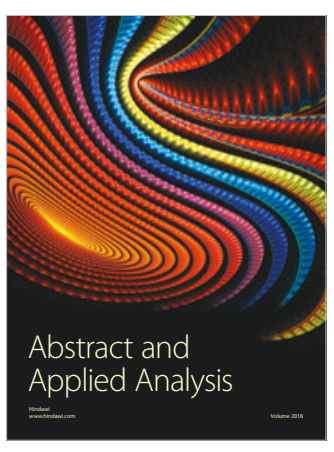

The Scientific

World Journal

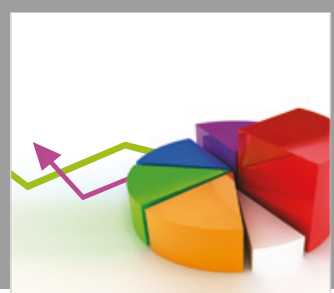

Journal of

Probability and Statistics
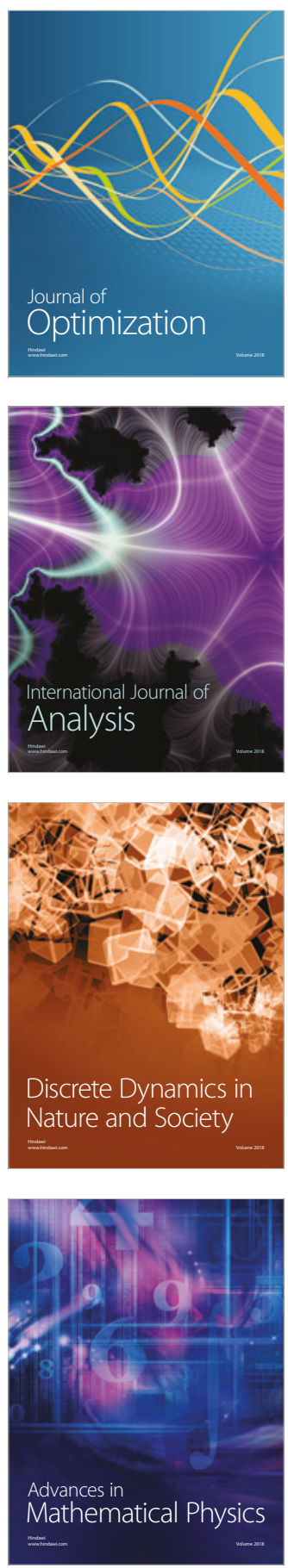